\title{
Effect of sodium-glucose linked transporter-2 inhibitors on heart failure end points in people with type 2 diabetes mellitus: a systematic review and meta-analysis
}

\author{
THOMAS SJ CRABTREE, 1,2 ROBERT EJ RYDER ${ }^{2}$
}

\begin{abstract}
Introduction: Type 2 diabetes is a condition which is frequently associated with macrovascular complications. Sodium-glucose linked transporter-2 inhibitors (SGLT2i) have been demonstrated to improve composite cardiovascular outcomes assessed via a 3-point Major Adverse Cardiovascular Events (MACE). Although they yield some benefit in reducing overall rates of cardiovascular death, stroke and myocardial infarction, it appears that the majority of the beneficial effects of SGLT2i drugs on composite outcomes are mediated by improvements in heart failure outcomes reducing cardiovascular death. This effect has been noted across multiple different drugs in the SGLT2i class. The aim of this review was to synthesise current evidence from randomised controlled trials (RCTs) comparing SGLT2i with placebo in adults with type 2 diabetes mellitus. The outcomes of interest were hazard ratios compared with placebo for hospitalisation due to heart failure (primary), death due to heart failure (secondary) and incidence rates of heart failure (secondary).

Methods: Searches were performed using recognised terms in MedLine, EMBASE, Pubmed, Cohrane CENTRAL and CINAHL. RCTs comparing SGLT2i with placebo were eligible for inclusion, providing they contained results for at least the outcome of interest. Studies were reviewed for inclusion by the two authors and data extraction and bias assessments were performed using a modified Cochrane's data extraction tool and bias assessment tool. Meta-analysis of hazard ratios (HRs) was performed in RevMan 5.4 using generic inverse variance and a fixed effects model.

Results: 3,212 records were identified of which 13 were eventually included, covering 11 clinical studies. The risk of hospi-
\end{abstract}

University Hospitals of Derby and Burton NHS Trust; University of Nottingham, Nottingham, UK

City Hospitals, Sandwell and West Birmingham Hospitals NHS Trust, Birmingham, UK

Address for correspondence: Dr Thomas SJ Crabtree Department of Diabetes, Royal Derby Hospital, Uttoxeter Road, Derby, DE22 3NE

E-mail: t.crabtree@nhs.net

https://doi.org/10.15277/bjd.2021.307 talisation for heart failure was significantly lower with SGLT2i compared to placebo (HR 0.69; $95 \% \mathrm{Cl} 0.64,0.74$ ). Inter-study heterogeneity was minimal $\left(I^{2}=0 \%\right)$ Only one study contained outcomes for death due to heart failure, but its results were not significant. No current studies report hazard ratios for heart failure diagnoses with SGLT2i use compared with placebo.

Conclusion: SGLT2i drugs reduce the rates of hospitalisation due to heart failure in people with type 2 diabetes. This may help mediate the improvements seen in composite cardiovascular outcomes. More evidence is needed to support their use in reducing mortality due to heart failure and incidence rates of new heart failure in this high-risk cohort.

Br J Diabetes 2021;21:186-191

Key words: diabetes, type 2, heart failure, SGLT-2, CVOT

\section{Introduction}

Diabetes is a condition associated with significant macrovascular risk. ${ }^{1}$ The incidence of heart failure among people with diabetes is significantly higher than in those without diabetes, especially at younger ages. ${ }^{2}$ Approximately $12 \%$ of people with type 2 diabetes are estimated to have heart failure. ${ }^{3}$ Although mortality due to heart failure has improved marginally, it remains poor compared with other life-limiting conditions such as cancer. ${ }^{4}$ People with diabetes and heart failure with reduced or preserved ejection fraction have worse outcomes than those without diabetes. ${ }^{5}$ Recent estimates place hospitalisation rates for heart failure in people with type 2 diabetes at 12.4 per 1,000 person/years compared with 2.4 per 1,000 person/years in those without diabetes. ${ }^{6}$

Until recently, diabetes therapies have shown limited efficacy in improving cardiovascular outcomes beyond any limited effect improvements in $\mathrm{HbA}_{1 c}$ may yield. Furthermore, some drugs such as rosiglitazone were associated with increases in adverse cardiovascular outcomes, particularly heart failure. ${ }^{7}$ Following the safety concerns surrounding rosiglitazone, new diabetes drugs were mandated to undergo robust trials to assess cardiovascular safety before approval, ${ }^{8}$ and this requirement continues. ${ }^{9}$

Sodium-glucose linked transporter-2 inhibitors (SGLT2i) were first introduced in the 2010s for the management of type 2 diabetes mellitus. Given the new mandate for cardiovascular outcome data prior to approval, phase III trials were designed to assess these 
outcomes using a 3-point Major Adverse Cardiovascular Events (MACE): a composite outcome of cardiovascular death, non-fatal myocardial infarction and stroke. ${ }^{10}$ Although heart failure is not part of the MACE end point, it has been assessed separately and may contribute to improved MACE by reducing cardiovascular death. The EMPA-REG trial was the first of these to report in 2015 and demonstrated reductions in mortality due to cardiovascular causes as well as MACE. ${ }^{11}$ Subsequent evidence has shown similar findings for other drugs in the class. ${ }^{12,13}$ Most notably, SGLT2i drugs seem to be beneficial in heart failure, with more recent trials supporting their use in people with heart failure irrespective of co-morbid diabetes, ${ }^{13-15}$ with improvements in cardiovascular mortality and heart failure hospitalisation rates. Early meta-analyses of five studies of SGLT2i showed improvements in rates of hospitalisation for heart failure with a pooled hazard ratio $(\mathrm{HR})$ of $0.68(05 \% \mathrm{Cl} 0.61$ to $0.76) .{ }^{16}$ Since this review, further studies have since been published. Given the significant benefits of SGLT2i on heart failure, it may be that reductions in cardiovascular death are mediated via improvements in heart failure outcomes. Some have suggested future assessments should include heart failure hospitalisation as a fourth point in the MACE outcome to better appreciate the benefit of these drugs on the condition. ${ }^{17}$

These data from randomised controlled trials (RCTs) are further supported by real-world evidence. One striking study compared SGLT2i with dipeptidylpeptidase-4 inhibitors and showed significant reductions in hospitalisation for heart failure and all-cause mortality with HRs of 0.69 (95\% Cl 0.61 to $0.77 ; p<0.0001)$ and 0.59 (95\% $\mathrm{Cl} 0.52$ to $0.67 ; p<0.0001)$, respectively. ${ }^{18}$

The exact mechanism of action is not clear and there are many suggested possibilities but is thought to be independent of glycaemic outcomes and the osmotic diuretic effect of these drugs. ${ }^{19}$ One suggested mechanism of action is attenuation of sodiumhydrogen exchanger activity, which is often increased in heart failure and contributes to diuretic resistance and fluid retention. ${ }^{19,20}$ Other mechanisms of action have also been suggested including effects on cardiac remodelling, left ventricular hypertrophy and decreasing oxidative stress. ${ }^{19,21}$

It seems plausible that many of the beneficial effects of SGLT2i drugs may be mediated through improvements in heart failure outcomes, both in people with and without type 2 diabetes. The aim of this review was to assess current RCT evidence reporting heart failure outcomes in adults with type 2 diabetes to see whether this assumption may hold true.

\section{Aims}

The aim of this systematic review is to assess the effect of SGLT2i drugs on heart failure outcomes in adults with type 2 diabetes mellitus in $\mathrm{RCTs}$. A summary of the population, intervention, comparison and outcomes (or PICO) model for this systematic review is shown in Table 1.

\section{Methods}

\section{Protocol and registration}

This systematic review and meta-analysis was prospectively registered with PROSPERO (registration number: CRD42020223256) and the reporting of this review has been undertaken in accordance with PRISMA guidelines. ${ }^{22}$ The search was performed on 14 March 2021.
Table 1. Summary of the PICO (population, intervention, comparison and outcome) model used for this systematic review and meta-analysis

\begin{tabular}{|c|c|}
\hline & Description \\
\hline P (Population) & Adults aged $\geq 18$ years \\
\hline I (Intervention) & $\begin{array}{l}\text { Treatment with any sodium-glucose linked } \\
\text { transporter- } 2 \text { inhibitor }\end{array}$ \\
\hline C (Comparison) & Placebo \\
\hline O (Outcomes) & $\begin{array}{l}\text { Primary: } \\
\text { Mortality due to heart failure } \\
\text { Secondary: } \\
\text { Admission to hospital due to heart failure } \\
\text { Incident diagnosis of heart failure }\end{array}$ \\
\hline
\end{tabular}

\section{Eligibility criteria}

Randomised controlled trials comparing SGLT2i with placebo in adults (aged $\geq 18$ years) with type 2 diabetes were eligible for inclusion. Relevant sub-analyses of studies containing a broader population were eligible. Other study designs were not considered. Included studies contained data for at least one of the outcomes of interest. Studies including those with other types of diabetes or including pregnant individuals or children were excluded. No exclusions were made on grounds of data or language.

The primary outcome of interest was hospitalisation due to heart failure. Death due to heart failure, and incident diagnosis of heart failure were both secondary outcomes.

\section{Data sources and search strategy}

EMBASE, MedLine, PubMed, Cochrane CENTRAL and the Cumulative Index to Nursing and Allied Health Literature (CINAHL) were searched electronically. An example of the search terms used in OVID: Medline is shown in Table 2. These terms were adapted for each database as required. Language and date limits were not applied.

\section{Study screening and selection}

Identified studies were imported into EndNote v9.3 for reference management. Following the removal of duplicate manuscripts, titles and abstracts were screened for relevance. Potentially

Table 2. Example of search terms for retrieval of studies, in this case from Ovid: Medline electronic database at http://ovidsp.ovid.com/

1. diabet*

2. "heart failure" OR "cardiac failure" OR (("left ventricular" OR LV OR systolic OR diastolic) ADJ2 impairment) OR HFpEF OR HFrEH OR CCF OR HF

3. ("sodium-glucose" OR "sodium glucose") ADJ3 transport*

4. SGLT2* OR "SGLT-2*" OR -gliflozin

5. 3 OR 4

6. 1 AND 3 AND 5 
relevant manuscripts were then reviewed in full-text against the inclusion and exclusion criteria before a decision was made regarding final inclusion. Review articles identified by the search were cross-referenced to ensure no potentially relevant studies were missed. All identified full-text articles were readily accessible for inclusion; no data or manuscript requests to authors were required on this occasion.

All studies were reviewed independently by two reviewers (TSCJ and REJR). No disputes occurred in performing this review; a third reviewer was available to adjudicate the inclusion of manuscripts/studies if needed.

\section{Data extraction}

Data were extracted using a modified Cochrane's data collection form for RCTs specific to the needs of this review ${ }^{23}$ and is included as an appendix to this manuscript (See Appendix 1 online at www.bjd-abcd.com). Key data to be extracted included the number of participants in each arm of the trial, the baseline characteristics of the study participants (weight, BMI, $\mathrm{HbA}_{1 c}$, age, ethnicity, duration of diabetes) and relevant co-morbidities including but not limited to pre-existing renal or cardiovascular disease. Outcomes were captured as the number of participants in each arm experiencing the outcome by the end of the study period. Bias assessment was performed using Cochrane's Collaboration bias assessment tool for RCTs. ${ }^{24}$

\section{Synthesis of results}

All data available for the primary outcome of hospitalisation due to heart failure in a suitable format, was incorporated into metaanalysis. Any studies which could not be included in meta-analysis have been synthesised narratively. Meta-analysis was conducted using generic inverse variance, log[hazard ratios] and standard error in RevMan 5.4 using a fixed-effects model and inter-study heterogeneity was assessed and reported using $R^{2}$ statistics. For the secondary outcomes of death due to heart failure or incident diagnosis of heart failure, a narrative synthesis was conducted due to limited avaialble data from the identified studies.

\section{Results}

A total of 3,210 records were identified, subsequently 1,581 duplicates immediately removed. The remaining 1,629 were screened, of which 89 were identified for full-text review following screening. Four additional studies of potential relevance were identified from cross-referencing pre-existing review articles. Eleven records covering nine clinical studies were eventually identified for inclusion in this review. Eight of these had data available in a format suitable for meta-analysis for hospitalisation due to heart failure; the other has been included in narrative form. At a later stage two further key studies were added following their presentation and publication (listed as other in the flow-chart). The review process is summarised in the flow chart shown in Figure 1. The characteristics of the identified studies are summarised in Table 3.

\section{Risk of bias assessment}

Bias assessment concluded potential sources of bias in most of the studies, although much of this was due to lack of clarity in
Figure 1. Flow chart showing the systematic review process and the numbers excluded at each stage

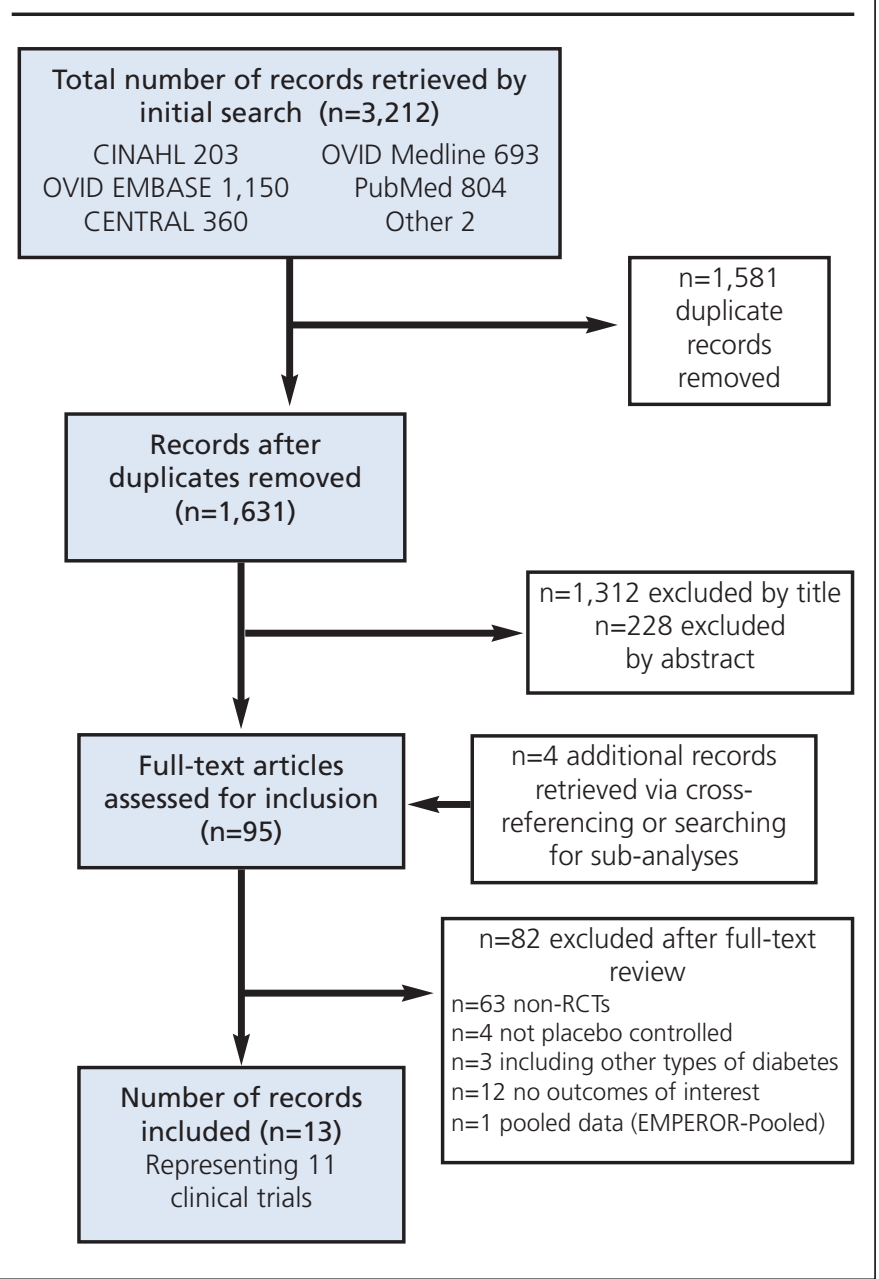

the reviewed manuscripts. The effect of potential bias introduced by changes in protocol mid-way through many of the studies is uncertain. This was the case with multiple studies, where adaptations were made in light of new published outcome data from other drugs in the class. On assessment, these protocol amendments were done in a valid way without unblinding or affecting the outcomes. Included studies with major protocol revisions for this reason have therefore be highlighted as having an "unclear" risk of bias in the "other bias" domain. The risk of bias assessment for this systematic review is summarised in Table 4.

\section{Primary outcome}

All of the identified studies had outcome data for hospitalisation due to heart failure. Ten of the studies were included in metaanalysis. One study, DAPA-CKD, ${ }^{25}$ did not report a HR for this outcome for the sub-group of people with type 2 diabetes and was therefore not included in the meta-analysis. However, data were available to calculate the number of events in the intervention and comparator arms during the follow-up period of the study, with 30 hospitalisations due to heart failure occurring in the intervention arm $(n=1,455)$ compared with 63 in the control 
Table 3. Summary of baseline characteristics and design of studies included in this review

\begin{tabular}{|c|c|c|c|c|c|c|c|c|c|}
\hline $\begin{array}{l}\text { Study name } \\
\text { or reference }\end{array}$ & Drug & $\begin{array}{l}\text { Study } \\
\text { region }\end{array}$ & $\begin{array}{l}\text { Number in } \\
\text { intervention } \\
\text { group, n/total, } \mathrm{N}^{\dagger}\end{array}$ & $\begin{array}{l}\text { Follow-up } \\
\text { duration, } \\
\text { median (weeks) }\end{array}$ & $\begin{array}{l}\text { Age } \\
\text { (years) } \\
\text { mean } \pm S D\end{array}$ & $\begin{array}{l}\text { Ethnicity } \\
\text { (\% white) }\end{array}$ & $\begin{array}{l}\mathrm{HbA}_{1 \mathrm{c}} \\
(\%)^{*} \\
\text { mean } \pm \mathrm{SD}\end{array}$ & $\begin{array}{l}\mathrm{BMI} \\
\left(\mathrm{kg} / \mathrm{m}^{2}\right) \\
\text { mean } \pm \mathrm{SD}\end{array}$ & $\begin{array}{l}\text { Outcomes of } \\
\text { interest (all due to } \\
\text { heart failure) }\end{array}$ \\
\hline CANVAS $^{25}$ & Canagliflozin & Global & $5,795 / 10,142$ & 126.1 & $63.3 \pm 8.3$ & $78.3 \%$ & $8.2 \pm 0.9$ & $32.0 \pm 5.9$ & Hospitalisation Death \\
\hline CREDENCE $^{26}$ & Canagliflozin & Global & $2,202 / 4,401$ & 136.8 & $63.0 \pm 9.2$ & $66.6 \%$ & $8.3 \pm 1.3$ & $31.3 \pm 6.2$ & Hospitalisation \\
\hline DAPA-CKD ${ }^{27} \ddagger$ & Dapagliflozin & Global & $1,455 / 2,906$ & 125.3 & $56.0 \pm 14.6$ & $54 \%$ & $5.6 \pm 0.4$ & $\mathrm{n} / \mathrm{a} 9$ & Hospitalisation \\
\hline DAPA-HF $28,29 *$ & Dapagliflozin & Global & $1,075 / 2,139$ & 79.2 & $66.3 \pm 9.9$ & $69.2 \%$ & $7.4 \pm 1.5$ & $29.4 \pm 6.1$ & Hospitalisation \\
\hline DECLARE-TIMI 5830* & Dapagliflozin & Global & $8,582 / 17,160$ & 219.2 & $63.9 \pm 6.8$ & $79.7 \%$ & $8.3 \pm 1.2$ & $32.1 \pm 6.0$ & Hospitalisation \\
\hline EMPA-REG $^{11 *}$ & Empagliflozin & Global & $4,687 / 7,020$ & 161.8 & $63.1 \pm 8.6$ & $72.6 \%$ & $8.1 \pm 0.9$ & $30.6 \pm 5.3$ & Hospitalisation \\
\hline EMPEROR-Preserved ${ }^{3 *} \infty$ & Empagliflozin & Global & $1,466 / 2,938$ & 113.4 & $71.8 \pm 9.3$ & $76.3 \%$ & Not reported & $29.8 \pm 5.8$ & Hospitalisation \\
\hline EMPEROR-Reduced ${ }^{14,15 *}$ & Empagliflozin & Global & $927 / 1,856$ & 69.6 & $66.8 \pm 10.0$ & $69.7 \%$ & $7.4 \pm 1.6$ & $28.8 \pm 5.5$ & Hospitalisation \\
\hline SCORED *34 & Sotagliflozin & Global & $5,292 / 10,584$ & 69.3 & $69(63-74) 3$ & $83.2 \%$ & $8.3(7.6-9.3)$ & $31.9(28.1-36.2)^{\S}$ & Hospitalisation \\
\hline SOLOIST ${ }^{31 *}$ & Sotagliflozin & Global & $608 / 1,222$ & 40 & $69(63-76)^{\S}$ & $93.2 \%$ & $7.2(6.4-8.2)^{\S}$ & $30.4(26.3-34.3)^{\S}$ & Hospitalisation \\
\hline VERTIS-CV 32 & Ertugliflozin & Global & $5,499 / 8,246$ & 182.7 & $64.4 \pm 8.1$ & $87.8 \%$ & $8.2 \pm 1.0$ & $31.9 \pm 5.4$ & Hospitalisation \\
\hline
\end{tabular}

Table 4. Risk of bias assessment for included randomised controlled trials (RCTs) using the Cochrane Risk of Bias assessment tool for randomised controlled trials ${ }^{24}$

\begin{tabular}{|c|c|c|c|c|c|c|c|c|c|c|c|}
\hline Bias Assessment - RCTs & CANVAS $^{25}$ & CREDENCE $^{26}$ & DAPA-CKD 27 & DAPA-HF ${ }^{28,29}$ & $\begin{array}{l}\text { DECLARE- } \\
\text { TIMI } 58^{30}\end{array}$ & $\begin{array}{l}\text { EMPEROR- } \\
\text { Reduced }^{14,15}\end{array}$ & $\begin{array}{l}\text { EMPA- } \\
\text { REG }^{11}\end{array}$ & SOLOIST ${ }^{31}$ & $\begin{array}{l}\text { VERTIS- } \\
\text { CV }^{32}\end{array}$ & SCORED $^{34}$ & $\begin{array}{l}\text { EMPEROR- } \\
\text { Preserved }^{33}\end{array}$ \\
\hline 1. Random sequence generation & Low & Low & Low & Low & Low & Low & Low & Low & Low & Low & Low \\
\hline 2. Allocation concealment & Low & Low & Low & Low & Low & Low & Low & Low & Low & Low & Low \\
\hline 3. Blinding of participants and personnel & Low & Low & Low & Low & Low & Low & Low & Low & Low & Low & Low \\
\hline 4. Blinding of outcome assessment & Unclear & Unclear & Low & Low & Unclear & Unclear & Unclear & Low & Unclear & Unclear & Unclear \\
\hline 5. Incomplete outcome data & Low & Low & Unclear & Low & Low & Low & Low & Unclear & Low & Low & Low \\
\hline 6. Selective reporting & Low & Low & Low & Unclear & Low & Low & Low & Unclear & Low & Unclear & Low \\
\hline 7. Other sources of bias & Unclear & Low & Low & Low & Unclear & Low & Unclear & Unclear & Low & Unclear & Unclear \\
\hline
\end{tabular}

arm $(n=1,451)$. The rate of hospitalisation due to heart failure with dapagliflozin was 2.1 per 100 person-years versus 3.8 per 100 person-years with placebo. One of the secondary outcomes for this study was a composite of cardiovascular mortality or hospitalisation due to heart failure and reported a HR of 0.7 (95\% $\mathrm{Cl} 0.52$ to 0.92 ).

The meta-analysis of the results from the other 10 trials is shown in Figure 2. The studies included 65,708 patients of which 36,133 were taking an SGLT2i drug. The pooled hazard ratio for hospitalisation due to heart failure was $0.69(95 \% \mathrm{Cl} 0.64,0.74)$ significantly favouring SGLT2i to placebo. Inter-study heterogeneity was very low with an $R^{2}$ value of $0 \%$. Of note, this is also comparable to the HRs for the composite outcome of hospitalisation due to heart failure or cardiac death in DAPA-CKD.

\section{Secondary outcomes}

Only one study reported mortality rates due specifically to heart failure. This study, the CANVAS study, reported a HR of 0.89 (95\% Cl 0.49 to 1.6 ) for canagliflozin compared with placebo. This fails to reach statistical significance. No studies reported the rates of incident new heart failure diagnoses with SGLT2i drugs compared with placebo.

\section{Discussion}

The cardiovascular outcome trials of SGLT2i drugs to date have provided robust and consistent evidence that their use in people with type 2 diabetes is associated with improved cardiac outcomes. In this systematic review looking specifically at heart failure outcomes, all identified studies favoured SGLT2i drugs to placebo (HR 0.69; 95\% Cl 0.64, 0.74) with very limited interstudy heterogeneity. This is comparable to the earlier meta-analysis of the five studies published at that point (HR 0.68; 0.61 to 0.76). ${ }^{16}$ This is strong evidence that SGLT2i drugs reduce the risk of hospital admission due to heart failure and that this is likely to be a class effect. Several of the studies feature a composite outcome of death due to cardiovascular causes and hospitalisation due to heart failure - the significance of SGLT2i drugs in reducing this outcome may be primarily mediated by reductions in hospitalisation due to heart failure.

Unfortunately, the available studies did not consistently report mortality rates due to heart failure. The one study with this outcome demonstrated a trend towards reduced mortality, but failed to reach statistical significance. Further studies or sub-analysis of current works is needed to establish whether SGLT2i drugs improve heart failure-specific mortality. 
Figure 2. Forrest plot showing meta-analysis of hazard ratios for hospitalisation due to heart failure for included studies

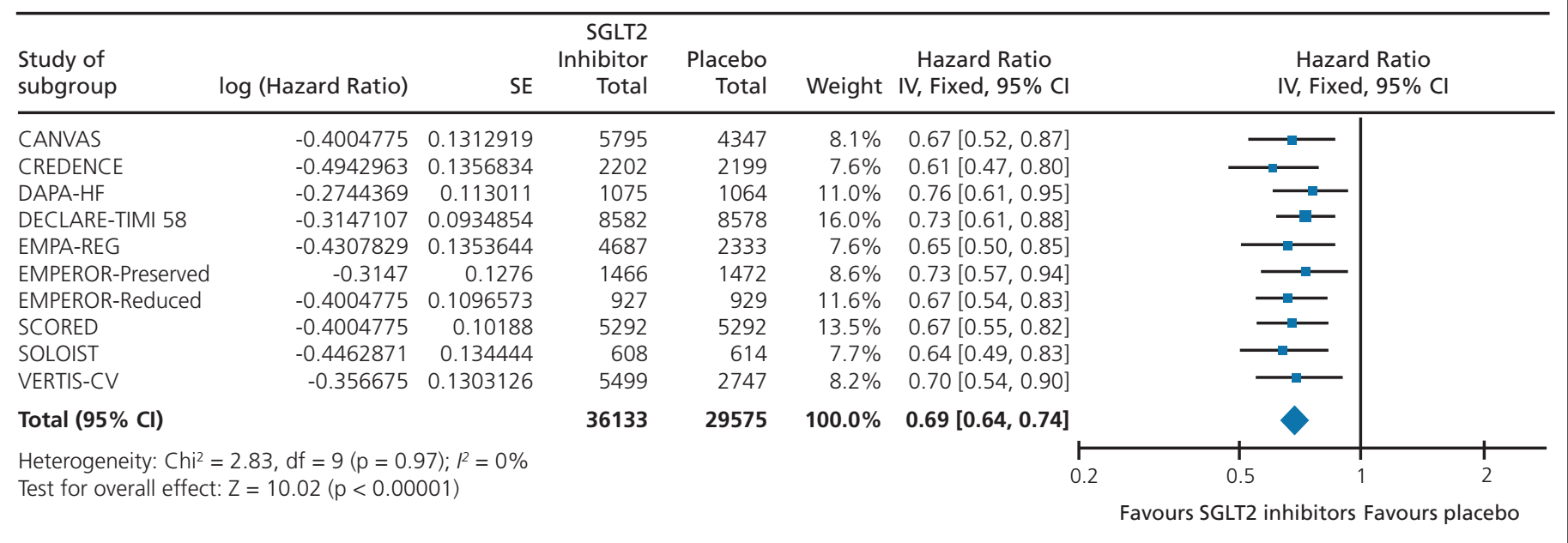

No studies reported the incident rates of new heart failure diagnoses. Most studies to date have been in people with significant cardiovascular risk factors. Further work is needed to assess incident heart failure diagnosis rates in those with these significant risk factors but without heart failure at baseline.

The paucity of data from studies for two of the outcomes limits the conclusions we are able to draw at present from this review. SGLT2i drugs appear to be well tolerated by most, but potential side effects including ketoacidosis and urinary tract infection may limit their use in some people with diabetes. ${ }^{35}$

Both SGLT2i and long acting GLP1-receptor agonists improve cardiovascular outcomes and they appear to provide benefit by entirely different mechanisms. ${ }^{19,36}$ Although SGLT2i drugs appear to reduce rates of cardiovascular disease and heart outcomes more than metformin, ${ }^{37}$ the cost difference between these drugs makes cost-effectiveness less certain. Further health-economic analyses will be needed to establish this moving forwards. Cardiovascular benefit is also well established for pioglitazone ${ }^{19}$ and it has been argued that SGLT2i, long-acting GLP-1 receptor agonists, pioglitazone and metformin could complement each other, if used in combination, to further improve cardiovascular outcomes. ${ }^{19}$ Nevertheless, the benefits of SGLT2i drugs in improving cardiovascular outcomes are clear, and we welcome their inclusion in the American Diabetes Association (ADA) and European Association for the Study of Diabetes (EASD) joint type 2 diabetes treatment algorithm - especially their use as second-line therapy in those at increased risk. ${ }^{38}$ Furthermore, we look forward to seeing their evolving role in the management of type 1 diabetes and future evidence that they improve cardiovascular outcomes beyond the positive glycaemic and weight outcomes demonstrated by the DEPICT trials. ${ }^{39}$

\section{Conclusion}

SGLT2i drugs significantly reduced heart failure hospitalisation rates in adults with type 2 diabetes in RCTs compared with placebo. This occurs, without any heterogeneity, in all drugs across the class in the studies identified for inclusion in this review. This may be the main mediator of improved composite

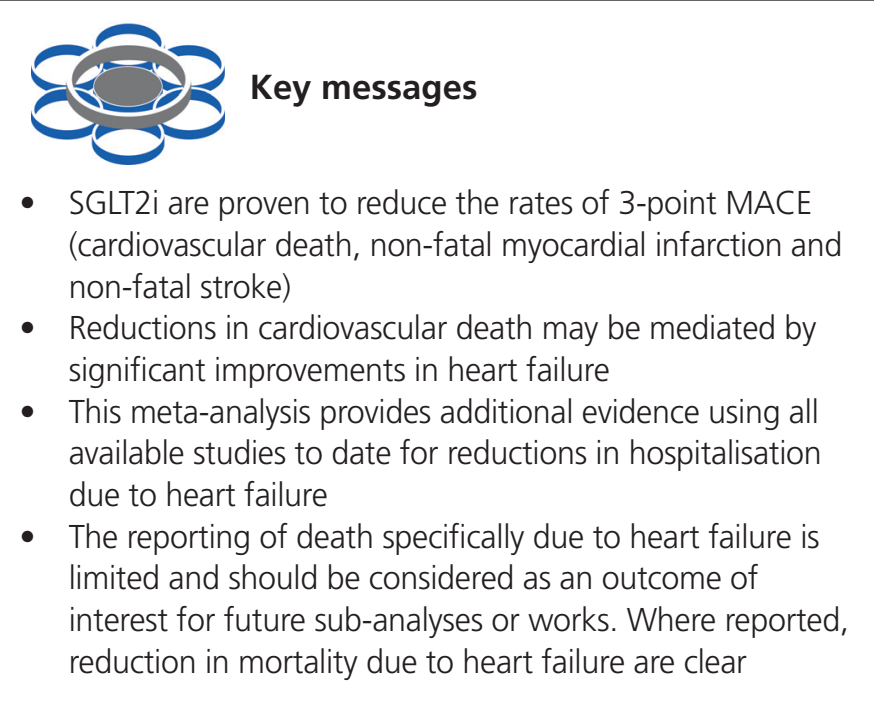

cardiovascular outcomes reported in many of the trials. It is not clear whether SGLT2i drugs reduce mortality rates due to heart failure or incident diagnosis. We eagerly await further work to clarify the benefits of SGLT2i drugs in this regard.

Conflict of interest TSJC has received speaker fees and educational support from Novo Nordisk and Sanofi. REJR has received speaker fees, and/or consultancy fees and/or educational sponsorships from AstraZeneca, BioQuest, GI Dynamics, Janssen and Novo Nordisk.

\section{Funding None.}

\section{References}

1. Sarwar N, Gao P, Kondapally SR, et al. Diabetes mellitus, fasting blood glucose concentration, and risk of vascular disease: a collaborative metaanalysis of 102 prospective studies. Lancet 2010;375(9733):2215-22. https://doi.org/10.1016/S0140-6736(10)60484-9

2. Nichols GA, Gullion CM, Koro CE, Ephross SA, Brown JB. The incidence of congestive heart failure in type 2 diabetes. Diabetes Care 2004; 27(8):1879-84. https://doi.org/10.2337/diacare.27.8.1879

3. Thrainsdottir IS, Aspelund T, Thorgeirsson G, et al. The association between glucose abnormalities and heart failure in the population-based 
Reykjavik study. Diabetes Care 2005;28(3):612-16. https://doi.org/ 10.2337/diacare.28.3.612

4. Taylor CJ, Ordóñez-Mena JM, Roalfe AK, et al. Trends in survival after a diagnosis of heart failure in the United Kingdom 2000-2017: population based cohort study. BMJ 2019;364:1223. https://doi.org/10.1136/ bmj.1223

5. MacDonald MR, Petrie MC, Varyani F, et al. Impact of diabetes on outcomes in patients with low and preserved ejection fraction heart failure: an analysis of the Candesartan in Heart failure: Assessment of Reduction in Mortality and morbidity (CHARM) programme. Eur Heart J 2008; 29(11):1377-85. https://doi.org/10.1093/eurheartj/ehn153

6. McAllister DA, Read SH, Kerssens J, et al. Incidence of hospitalization for heart failure and case-fatality among 3.25 million people with and without diabetes mellitus. Circulation 2018;138(24):2774-86. https://doi.org/ 10.1161/CIRCULATIONAHA.118.034986

7. Wallach JD, Wang K, Zhang AD, et al. Updating insights into rosiglitazone and cardiovascular risk through shared data: individual patient and summary level meta-analyses. BMJ 2020;368:I7078. https://doi.org/ $10.1136 / \mathrm{bmj} .17078$

8. Bae JC. Diabetes drugs and cardiovascular safety. Endocrinol Metab (Seoul) 2016;31(2):239-44. https://doi.org/10.3803/EnM.2016.31.2.239

9. Low Wang CC, Everett BM, Burman KD, Wilson PWF. Cardiovascular safety trials for all new diabetes mellitus drugs? Circulation 2019;139(14):17413. https://doi.org/10.1161/CIRCULATIONAHA.118.038771

10. Zinman B, Inzucchi SE, Lachin JM, et al. Rationale, design, and baseline characteristics of a randomized, placebo-controlled cardiovascular outcome trial of empagliflozin (EMPA-REG OUTCOMETM). Cardiovasc Diabeto/ 2014;13(1):102. https://doi.org/10.1186/1475-2840-13-102

11. Zinman B, Wanner C, Lachin JM, et al. Empagliflozin, cardiovascular outcomes, and mortality in type 2 diabetes. N Engl J Med 2015; 373(22):2117-28. https://doi.org/10.1056/NEJMoa1504720

12. Cannon $C P$, Perkovic $V$, Agarwal $R$, et al. Canagliflozin reduces cardiovascular and renal events in patients with type 2 diabetes and chronic kidney disease regardless of baseline $\mathrm{HbA} 1 \mathrm{c}$, including those with $\mathrm{HbA} 1 \mathrm{c}<7 \%$ : results from the CREDENCE trial. Circulation 2019;141(5):407-10. https://doi.org/10.1161/CIRCULATIONAHA.119.044359

13. Bhatt DL, Verma S, Braunwald E. The DAPA-HF trial: a momentous victory in the war against heart failure. Cell Metab 2019;30(5):847-9. https://doi.org/10.1016/j.cmet.2019.10.008

14. Anker SD, Butler J, Filippatos $G$, et al. Effect of empagliflozin on cardiovascular and renal outcomes in patients with heart failure by baseline diabetes status: results from the EMPEROR-Reduced trial. Circulation 2021; 143(4)337-49. https://doi.org/10.1161/CIRCULATIONAHA.120.051824

15. Packer M, Anker SD, Butler J, et al. Cardiovascular and renal outcomes with empagliflozin in heart failure. N Engl J Med 2020;383(15):141324. https://doi.org/10.1056/NEJMoa2022190

16. McGuire DK, Shih WJ, Cosentino F, et al. Association of SGLT2 inhibitors with cardiovascular and kidney outcomes in patients with type 2 diabetes: a meta-analysis. JAMA Cardio/ 2021;6(2):148-58. https://doi.org/ 10.1001/jamacardio.2020.4511

17. Hupfeld C, Mudaliar S. Navigating the "MACE" in cardiovascular outcomes trials and decoding the relevance of atherosclerotic cardiovascular disease benefits versus heart failure benefits. Diabetes Obes Metab 2019:21(8):1780-9. https://doi.org/10.1111/dom.13740

18. Kohsaka S, Lam CSP, Kim DJ, et al. Risk of cardiovascular events and death associated with initiation of SGLT2 inhibitors compared with DPP4 inhibitors: an analysis from the CVD-REAL 2 multinational cohort study. Lancet Diabetes Endocrinol 2020;8(7):606-15. https://doi.org/10.1016/ S2213-8587(20)30130-3

19. Ryder, RE, MA Abdul-Ghani, RA DeFronzo. Diabetes medications with cardiovascular protection: the likelihood of benefit from combination therapy increases further following new evidence during 2020. $\mathrm{Br} J$ Diabetes 2020;20(2):84-88. https://doi.org/10.15277/bjd.2020.276

20. Packer M, Anker SD, Butler J, Filippatos G, Zannad F. Effects of sodiumglucose cotransporter 2 inhibitors for the treatment of patients with heart failure: proposal of a novel mechanism of action. JAMA Cardiol 2017;2(9):1025-9. https://doi.org/10.1001/jamacardio.2017.2275

21. Lopaschuk GD, Verma S. Mechanisms of cardiovascular benefits of sodium glucose co-transporter 2 (SGLT2) inhibitors: a state-of-the-art review. JACC Basic Transl Sci 2020;5(6):632-44. https://doi.org/ 10.1016/j.jacbts.2020.02.004

22. Moher D, Liberati A, Tetzlaff J, Altman DG, PRISMA Group. Preferred reporting items for systematic reviews and meta-analyses: the PRISMA statement. BMJ 2009;339:b2535. https://doi.org/10.1136/bmj.b2535

23. Higgins JPT, Chandler J, Cumpston M, Li T, Page MJ, Welch VA (eds). Cochrane Handbook for Systematic Reviews of Interventions version 6.1. 2020. www.training. cochrane.org/handbook

24. Higgins JPT, Altman DG, Gøtsche PC, et al. The Cochrane Collaboration's tool for assessing risk of bias in randomised trials. BMJ 2011;343:d5928. https://doi.org/10.1136/bmj.d5928

25. Neal B, Perkovic V, Mahaffey KW, et al. Canagliflozin and cardiovascular and renal events in type 2 diabetes. N Engl J Med 2017:377(7):644-57. https://doi.org/10.1056/NEJMoa1611925

26. Perkovic V, Jardine MJ, Neal B, et al. Canagliflozin and renal outcomes in type 2 diabetes and nephropathy. N Engl J Med 2019:380(24):2295306. https://doi.org/10.1056/NEJMoa1811744

27. Wheeler DC, Stefánsson BV, Jongs N, et al. Effects of dapagliflozin on major adverse kidney and cardiovascular events in patients with diabetic and non-diabetic chronic kidney disease: a prespecified analysis from the DAPA-CKD trial. Lancet Diabetes Endocrinol 2021;9(1):22-31. https://doi.org/10.1016/S2213-8587(20)30369-7

28. McMurray JJV, Solomon SD, Inzucchi SE, et al. Dapagliflozin in patients with heart failure and reduced ejection fraction. N Engl J Med 2019; 381(21):1995-2008. https://doi.org/10.1056/NEJMoa1911303

29. Petrie MC, Verma S, Docherty KF, et al. Effect of dapagliflozin on worsening heart failure and cardiovascular death in patients with heart failure with and without diabetes. JAMA 2020;323(14):1353-68. https://doi.org/10.1001/jama.2020.1906

30. Wiviott SD, Raz I, Bonaca MP, et al. Dapagliflozin and cardiovascular outcomes in type 2 diabetes. N Engl J Med 2019;380(4):347-57. https://doi.org/10.1056/NEJMoa1812389

31. Bhatt DL, Szarek M, Steg PG, et al. Sotagliflozin in patients with diabetes and recent worsening heart failure. N Engl J Med 2021;384(2):117-28. https://doi.org/10.1056/NEJMoa2030183

32. Cannon CP, Pratley R, Dagogo-Jack S, et al. Cardiovascular outcomes with ertugliflozin in type 2 diabetes. N Engl J Med 2020;383(15):142535. https://doi.org/10.1056/NEJMoa2004967

33. Anker SD, Butler J, Filippatos G et al. Empagliflozin in heart failure with a preserved ejection fraction. N Engl J Med 2021. https://doi.org/ 10.1056/NEJMoa2107038

34. Bhatt DL, Szarek M, Pitt B, et al. Sotagliflozin in patients with diabetes and chronic kidney disease. N Engl J Med 2020;384(2):129-139. https://doi.org/10.1056/NEJMoa2030186.

35. Singh $M$, Kumar A. Risks associated with SGLT2 inhibitors: an overview. Curr Drug Saf 2018;13(2):84-91. https://doi.org/10.2174/ 1574886313666180226103408

36. Kristensen SL, Rørth R, Jhund PS, et al. Cardiovascular, mortality, and kidney outcomes with GLP-1 receptor agonists in patients with type 2 diabetes: a systematic review and meta-analysis of cardiovascular outcome trials. Lancet Diabetes Endocrinol 2019;7(10):776-85. https://doi.org/ 10.1016/S2213-8587(19)30249-9

37. Eurich DT, Weir DL, Majumdar SR, et al. Comparative safety and effectiveness of metformin in patients with diabetes mellitus and heart failure: systematic review of observational studies involving 34,000 patients. Circ Heart Fail 2013;6(3):395-402. https://doi.org/10.1161/CIRCHEARTFAILURE.112.000162

38. Davies MJ, D'Alessio DA, Fradkin J, et al. Management of hyperglycemia in type 2 diabetes, 2018. A consensus report by the American Diabetes Association (ADA) and the European Association for the Study of Diabetes (EASD). Diabetes Care 2018;41(12):2669-701. https://doi.org/ 10.2337/dci18-0033

39. Dandona P, Mathieu C, Phillip M, et al. Efficacy and safety of dapagliflozin in patients with inadequately controlled type 1 diabetes: the DEPICT-1 52-Week Study. Diabetes Care 2018;41(12):2552-9. https://doi.org/10.2337/dc18-1087 
Appendix 1. Data extraction form

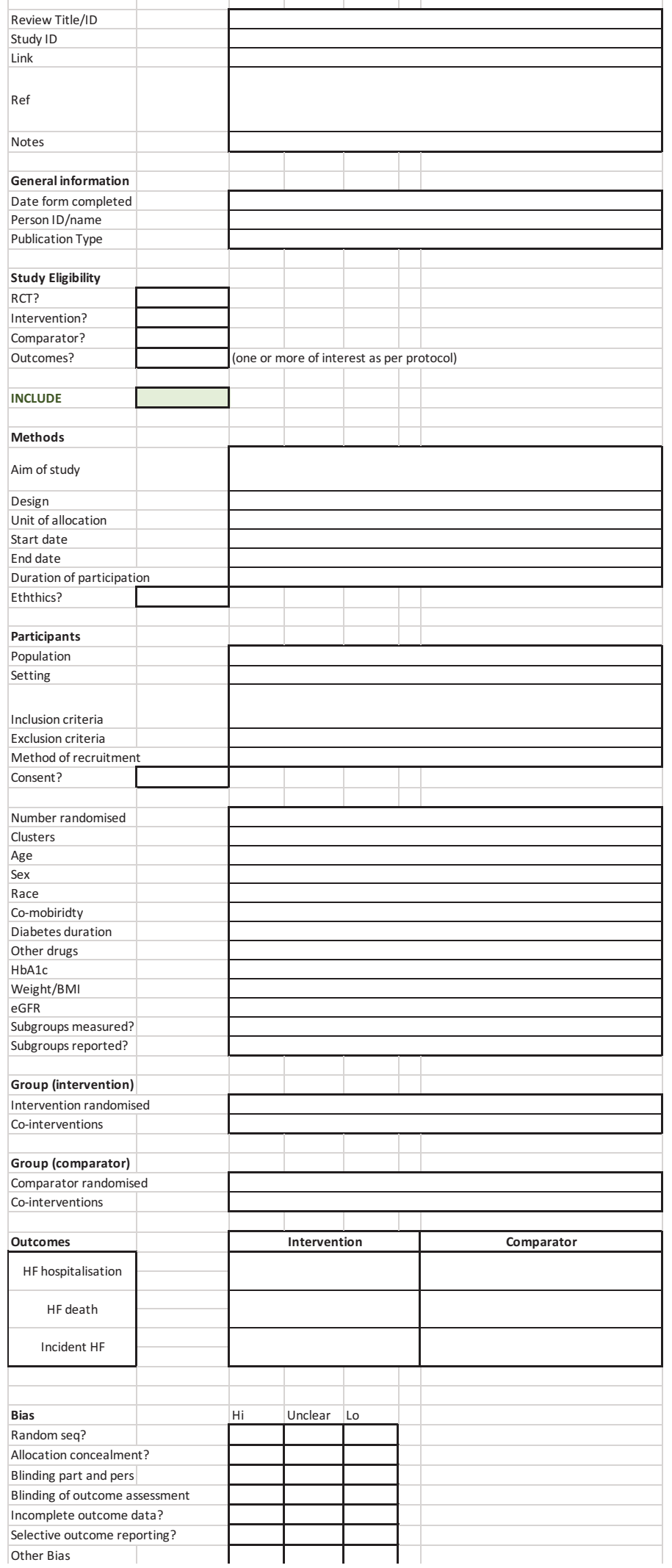

